\title{
What is colitis? Statistical approach to distinguishing clinically important inflammatory change in rectal biopsy specimens
}

\author{
D JENKINS, * A GOODALL,$\dagger$ K DREW $\ddagger$ B B SCOTT $\dagger$
}

From the *Department of Pathology, Whittington Hospital, the $\ddagger$ Computer Unit, School of Medicine, University College, London, and the †Department of Medicine, County Hospital, Lincoln

SUMMARY Measurements of mucosal dimension, architecture, and cell counts in both lamina propria and epithelium were made on rectal biopsy specimens from 20 patients with irritable bowel syndrome ("normal" controls); 54 patients with ulcerative colitis, Crohn's disease, and non-specific proctitis; eight patients with small bowel Crohn's disease; and 34 in whom the rectal biopsy specimen was not diagnostic. Discriminant analysis was applied to multiple variables based on the measurements, and three variables were identified as of high predictive value. The most powerful discriminant was increased lamina propria cellularity in all forms of chronic colitis. The ratios of surface length to mucosal length and of surface epithelial height to crypt epithelial height also $\vec{\bullet}$ emerged as discriminants. Chronic inflammatory bowel disease was distinguished from normal in $\stackrel{\infty}{\infty}$ $95 \%$ of cases with a definite pathological diagnosis, and $85 \%$ of borderline cases were correctly classified as either normal or inflammatory when judged by the final diagnosis after follow up.

This study provides a basis for automated diagnosis of rectal biopsy specimens and provides objectively validated criteria which can also be applied in routine histological diagnosis.

Sigmoidoscopy and biopsy form the mainstay of the investigation of suspected inflammatory bowel disease. Usually the results of sigmoidoscopy and biopsy concur about normality or abnormality of the rectal mucosa, but in about $5 \%$ of patients with ulcerative colitis and in $50 \%$ with Crohn's colitis the rectum will appear normal at sigmoidoscopy.' Diagnosis then depends entirely on histological judgment about the degree of inflammatory change in the biopsy specimen. There may be definitive changes of inflammatory bowel disease, but in many of these cases the problem is the importance of possible borderline changes in the number and distribution of normally present lymphocyte, plasma cell, and histiocyte populations, which are often routinely reported as "a mild increase in chronic inflammatory cells."

In the absence of any other evidence of inflammatory bowel disease the interpretation of these apparent, minimal histological changes, and the decision whether to begin treatment for inflammatory

Accepted for publication 9 September 1987 bowel disease can be very difficult. Although the biopsy features of acute self limited colitis and inflammatory bowel disease are well described and criteria for distinguishing between them have been validated statistically, ${ }^{2}$ the minimal criteria sufficient to justify a diagnosis of inflammatory disease on a rectal biopsy specimen are not clearly defined. Despite this, clinical importance has been attached to certain minor histological abnormalities. "Minimal change colitis" has been described in Crohn's disease and also ulcerative colitis. ${ }^{3}$ A group of patients with mild "microscopic colitis," normal sigmoidoscopy and radiological findings has been described as having severe watery diarrhoea responding to anti-inflammatory drugs. ${ }^{4}$ Chronic inflammation in rectal biopsy specimens from homosexuals has been associated with syphilis, herpes, and chlamydial infection. ${ }^{5}$

In a previous study we reported counts of rectal mucosal plasma cells in a normal control group and in patients with inflammatory bowel disease. ${ }^{6}$ In a study of duodenitis, we also showed that statistical techniques of classification, cluster analysis, and discriminant analysis could be applied to counts of epithelial 
and lamina propria inflammatory cell populations and to measurements of mucosal volume and architecture. ${ }^{7}$ These could be used to define statistically the quantitative histological features of normal mucosa and identify those features which could best distinguish clinically important inflammatory change.

For this study, measurements of cell populations and their distribution, and of mucosal volume and architecture, were made on rectal biopsy specimens from groups of patients with irritable bowel syndrome (normal controls) (A), known ulcerative colitis (B), non-specific proctitis (C), Crohn's colitis (D), and from patients with Crohn's disease of the small bowel (E), and a group with an apparent borderline "mild increase in chronic inflammatory cells" who were also subjected to clinical follow up (F). A stepwise discriminant analysis was used to derive discriminant functions for groups A, B, C, D and B to D combined. These were then used to study the classification of specimens from groups $E$ and $F$.

\section{Patients and methods}

\section{GROUP A}

This group of controls comprised 20 patients with diarrhoea, (eight men, 12 women; mean age 38 years, range 20-64 years), who after investigation were considered to have the irritable bowel syndrome and who had no evidence of organic gastrointestinal disease.

\section{GROUP B}

Twenty patients with diarrhoea were included in this group (nine men, 11 women; mean age 47 years, range 27-76 years). All had sigmoidoscopic appearances typical of ulcerative colitis and a barium enema showing colonic disease above the sigmoid colon. In 15 patients disease was apparently confined to the left side of the colon and in five it extended to the right side. The mean duration of disease was four years. Sixteen were taking sulphasalazine and none was taking steroids at the time of biopsy.

\section{GROUP C}

This group comprised 14 patients with diarrhoea (eight men, six women; mean age 35 years, range 19-74 years). All had typical sigmoidoscopic appearances of proctitis. There was either an upper limit of disease seen at rigid sigmoidoscopy or no evidence of colonic disease on barium enema. The mean duration of disease was four years. Six were taking sulphasalazine and none was taking steroids at the time of biopsy.

\section{GROUP D}

Nineteen patients with diarrhoea (13 men and six women; mean age 40 years, range $18-70$ years) were included. Crohn's disease was diagnosed mainly on the radiological evidence of small bowel disease in nine patients, discontinuous disease in one patient, typical endoscopic appearances in three patients, examination of the resected colon in four patients, and typical conventional histology on rectal biopsy in three patients. The mean duration of disease was four years. Eleven were taking sulphasalazine and none was taking steroids at the time of biopsy.

\section{GROUP E}

In this group were eight patients (six men and two women; mean age 41 years, range 19-61 years). All had radiological evidence of small bowel Crohn's disease and no evidence of disease of the colon or rectum on sigmoidoscopy and barium enema. None was taking sulphasalazine or steroids at the time of biopsy.

\section{GROUP F}

Thirty four patients ( 17 men and 17 women; mean age 45 years, range 17-81 years) whose rectal biopsy specimens were reported as showing a mild increase in chronic inflammatory cells but no other features suggestive of inflammatory bowel disease were included. Sigmoidoscopic appearances were normal. Ten had definite inflammatory bowel disease, including two in long term remission on treatment and one with Crohn's colitis not affecting the rectum on sigmoidoscopy. Twenty had no evidence of inflammatory bowel disease after full investigation and remained free of disease after follow up. Four patients were still being followed up for possible inflammatory bowel disease at the time of writing, but had not developed detectable disease.

Rectal biopsy specimens were taken during the course of routine management using Brock's biopsy forceps. The specimens were immediately fixed in formalin, and only those which were well oriented and of adequate size were used.

The fixed specimens were embedded into paraplast. Tissue sections were cut at $1 \mu$ thickness perpendicular to the luminal surface, mounted on glass slides, and dried overnight at $37^{\circ} \mathrm{C}$. Sections were stained with haematoxylin and eosin for routine histological examination, counts of total cellularity and polymorphonuclear neutrophils, and for architectural measurements. For plasma cell counts, sections were stained for $\operatorname{IgA}$, IgM, and IgG by the peroxidaseantiperoxidase-immunoperoxidase technique after initial trypsinisation and counterstained with Harris's haematoxylin. The method has been described previously. ${ }^{6}$

MEASUREMENTS

Measurements and cell counts on the stained sections 
were carried out using a MOPPET image analyser. An image of the stained section was projected at standard magnification on to the digitising tablet by a Leitz Neopromar projection microscope. The MOP stylus was fitted with a ballpoint pen to provide a permanent record of the field examined. A field was defined as the area between the mucosal surface and muscularis mucosae bounded by two lines perpendicular to the muscularis mucosae. Four contiguous, non-overlapping fields were examined on each section, with close alignment to ensure that measurements of architectural features and cell counts could be made over a minimum of at least one millimetre in mucosal length and, in most cases, over two to three millimetres of mucosa. A micrometer slide was used to convert area and length measurements on the projected image into absolute measurements. All processing, measurements, and counts were performed blind to minimise experimental and observer bias. To measure variation in architectural features and cell density the area of the four adjacent fields was divided vertically and horizontally by grid lines. Two grid lines parallel to the smoothed muscularis mucosae were drawn at one third and two thirds of the mean mucosal height. The mucosal length was divided and 11 equally placed straight lines were drawn perpendicular to the muscularis mucosae. The following measurements were made on the contiguous fields in each section (fig 1). Mucosal length was measured as the smoothed length in millimetres of the muscularis mucosae along its mucosal edge.

Surface length was measured as the smoothed length in millimetres of the surface epithelium measured along

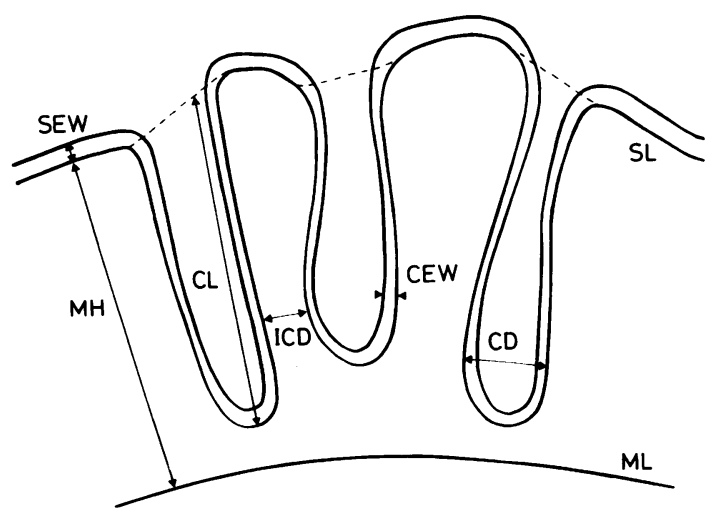

Fig 1 Measurements made on each section. $M L=$ mucosal length; $S L=$ surface length;

$M H=$ mucosal height $; C L=$ crypt length; $C D=$ crypt maximum diameter; $I C D=$ intercrypt distance; $S E W=$ surface epithelial width; $C E W=$ crypt epithelial width. the basement membrane, and interpolated across $\square$ mouths of crypts.

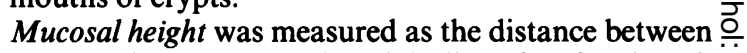
the line of mucosal length and the line of surface length in micrometres, perpendicular to the muscularis $\stackrel{\oplus}{+}$ mucosae along 11 equally spaced grid lines.

Cross sectional area was measured as the area in square $\frac{\overline{ }}{\bar{N}}$ millimetres of the whole mucosa between the lines of $\frac{\bar{D}}{\bar{\alpha}}$ mucosal length and surface length.

Lamina propria area was measured as the area in square millimetres of the lamina propria, excluding $\vec{\circ}$ crypts.

Crypt length was measured as the distance in $\vec{\omega}$ micrometres at right angles to the muscularis mucosae $\stackrel{\text { ? }}{\text {. }}$ between the basement membrane at the lowest point 웅 for each crypt and the basement membrane of the $A$ surface epithelium. Between 10 and 25 crypts were measured for each case.

Crypt maximum diameter was measured as the max- $\circ$ imum diameter in micrometres of each crypt between basement membranes, parallel to the muscularis $\vec{c}$ mucosae.

Intercrypt distance was measured as the least distance in micrometres between basement membranes of each $₹$ pair of adjacent crypts parallel to the muscularis $\vec{\varphi}$ mucosae.

Surface epithelial width was measured as the height of the surface cell in micrometres perpendicular to the basement membrane where the vertical grid lines cut the surface epithelium.

Crypt epithelial width was measured as the height of $\frac{0}{\mathbb{D}}$ the crypt epithelial cell in micrometres perpendicular $\stackrel{\Omega}{\Rightarrow}$ to the crypt basement membrane at the maximum horizontal diameter.

Counts were made of: polymorphs in surface epithelium, crypt epithelium, crypt lumina, and lamina propria; IgA, IgM and IgG plasma cells in the lamina propria; and total lamina propria cellularity excluding recognisable epithelial and smooth muscle cells. Table 3 . 1 shows the variables computed and entered into the $\delta$ discriminant analysis from these measurements and $₹$ cell counts.

All analyses were carried out on the Amdahl 470V/8 computer at the University of London Computer을. Centre. Discriminant analysis was performed using the computer package BMDP (University of Califor-s nia, 1985), programme BMDP7M. This computes a 0 linear classification for each of the selected groups in a N stepwise manner. At each step the variable which addso most to the separation of the groups is entered into the function; selected variables may also be forced into the function. The discriminants identified were then used $\stackrel{\infty}{\rightarrow}$ to classify the known and unknown groups using a "jack-knife" procedure to simulate a separate data set. The analyses were carried out between group $A$ and $\overparen{\Omega}$ groups B-D separately, and between normal and 
Table 1 Variables computed and entered into discriminant analysis

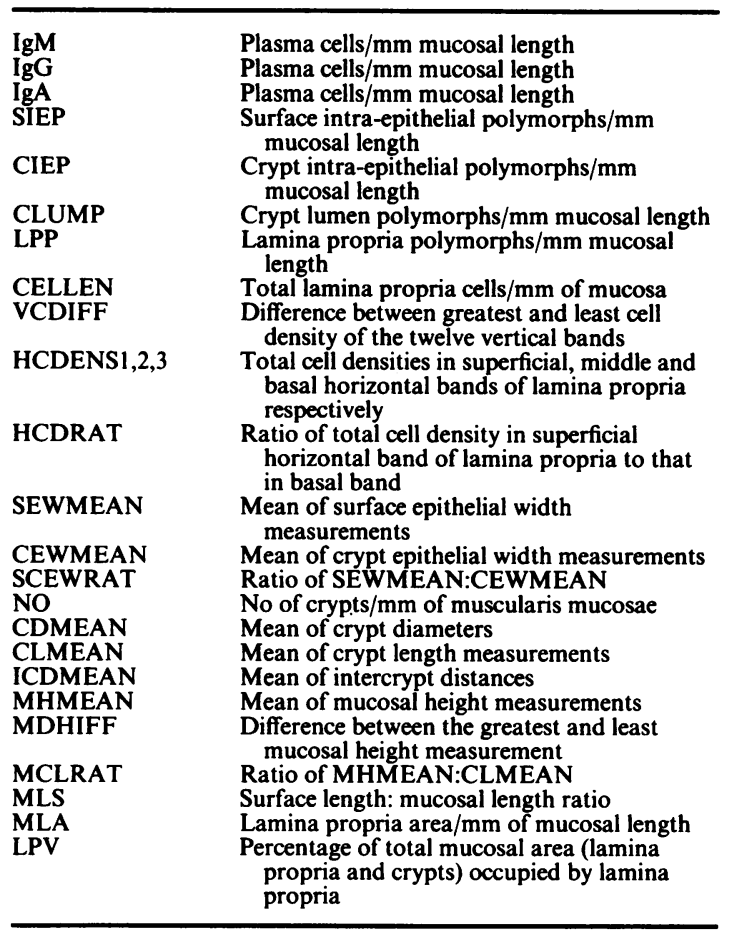

combined chronic inflammatory bowel disease as a single group. The analyses were carried out both including and excluding plasma cell counts obtained using the immunoperoxidase technique. The program only uses cases with complete data for the variables being used.

\section{Results}

Table 2 shows the mean values of the cell population variables entered for each group; table 3 the mean value of the mucosal measurement variables entered for each of the same groups. Total cellularity used on its own as a discriminant correctly classified $86 \%$ of biopsy specimens of known, normal, and chronic inflammatory bowel disease. Particular attention was therefore paid to obtaining an optimal discriminant for cellularity. The cell densities in horizontal bands in the lamina propria in the groups are shown in table 4. In group A there was a high concentration of lamina propria cells beneath the surface. In all forms of chronic inflammatory bowel disease cellularity was increased throughout the lamina propria, but there was also a change in the distribution of lamina propria cells, resulting in a very large increase in cellularity of the basal zone adjacent to the muscularis mucosae (HCDENS3), and a significant change in the ratio of cell density between the superficial and basal zones from about 1.8:1 in the normal state to about 1:1.

The jack-knifed results of stepwise discriminant analysis of the group A against combined inflammatory bowel disease, with and without immunoperoxidase plasma cell counts, are shown in table 5, together with the results of the classification of all the other groups. The variables entered were the same whether or not plasma cell counts were included. The most significant variable to emerge was the ratio of cell density between the superficial and deep parts of the lamina propria (HCDRAT). The ratio of surface epithelial height to crypt epithelial height (SCEWRAT) also emerged as a discriminant, as did the ratio of surface epithelial length to mucosal length (MLS). With these variables about $95 \%$ of known cases could be classified accurately.

Discriminant analysis was also performed between the normal controls and the groups of inflammatory bowel disease separately. With stepwise discriminant analysis between normal controls and patients with ulcerative colitis the same three discriminants emerged as in the combined analysis, whether plasma cell counts were included or not, the most important discriminant again being HCDRAT. Again, $95 \%$ of cases could be classified accurately using these three discriminants. When plasma cell counts were included, IgG plasma cell count was added as an additional discriminant, but this did not produce any overall improvement in accuracy of classification. Analysis of the normal group compared with those with Crohn's colitis showed that HCDRAT and SCEWRAT were the two best discriminants, with IgM plasma cell count as an additional discriminant. MLS approached threshold value as a discriminant with an F-to-enter of 3.85 (against a threshold set at 4.0). Both with and without the plasma cell count all cases were accurately classified after the jack-knife procedure. In group C total cellularity (CELLEN), together with mean distance between crypts (ICDMEAN), crypt lumen polymorphs (CLUMP), and SCEWRAT were entered when all variables were used. On jack-knife classification this produced a $97 \%$ accuracy in classification overall. When CELLEN was excluded, IgA, SCEWRAT, HCDRAT, CLUMP and IgG were entered and again there was $97 \%$ accuracy. When CELLEN and plasma cell counts were excluded, HCDRAT and SCEWRAT were best discriminants (100\% accurate classification of groups $A$ and $C)$.

When the discriminants HCDRAT, SCEWRAT, and MLS were used to classify the group E, all were identified as normal. In group $F 10$ cases were classified as inflammatory bowel disease and 24 as normal. When these cases were examined in relation to 
Table 2 Group means of mucosal counts

\begin{tabular}{|c|c|c|c|c|c|}
\hline Variable & Controls & $\begin{array}{l}\text { Ulcerative } \\
\text { colitis }\end{array}$ & $\begin{array}{l}\text { Chronic Crohn's } \\
\text { disease }\end{array}$ & Proctitis & $\begin{array}{l}\text { Small bowel } \\
\text { Crohn's disease }\end{array}$ \\
\hline $\begin{array}{l}\text { IgM } \\
\text { IgG } \\
\text { IgA } \\
\text { SIEP } \\
\text { CIEP } \\
\text { CLUMP } \\
\text { LPP } \\
\text { CELLEN }\end{array}$ & $\begin{array}{r}98.0 \\
40.8 \\
257.2 \\
6.0 \\
1.6 \\
0.6 \\
13.5 \\
1449.0\end{array}$ & $\begin{array}{r}338.7 \\
374.8 \\
731 \cdot 8 \\
10.0 \\
33.8 \\
4.1 \\
211 \cdot 3 \\
3158.8\end{array}$ & $\begin{array}{r}309 \cdot 7 \\
414 \cdot 0 \\
736 \cdot 0 \\
7 \cdot 7 \\
37 \cdot 4 \\
48 \cdot 7 \\
185 \cdot 4 \\
3505 \cdot 8\end{array}$ & $\begin{array}{r}262 \cdot 6 \\
605 \cdot 2 \\
980 \cdot 1 \\
10 \cdot 0 \\
52 \cdot 5 \\
46 \cdot 5 \\
314 \cdot 2 \\
5322 \cdot 8\end{array}$ & $\begin{array}{r}88.4 \\
46.6 \\
362.0 \\
6.6 \\
2.6 \\
0.4 \\
16.6 \\
1587.0\end{array}$ \\
\hline
\end{tabular}

Table 3 Group means of mucosal measurements

\begin{tabular}{|c|c|c|c|c|c|}
\hline Variable & Controls & $\begin{array}{l}\text { Ulcerative } \\
\text { colitis }\end{array}$ & $\begin{array}{l}\text { Chronic Crohn's } \\
\text { disease }\end{array}$ & Proctitis & $\begin{array}{l}\text { Small bowel } \\
\text { Crohn's disease }\end{array}$ \\
\hline $\begin{array}{l}\text { NO } \\
\text { MLS } \\
\text { MLA } \\
\text { LPV } \\
\text { CDMEAN } \\
\text { MHMEAN } \\
\text { SEWMEAN } \\
\text { CEWMEAN } \\
\text { CLMEAN } \\
\text { ICDMEAN } \\
\text { MHDIFF } \\
\text { VCDIFF } \\
\text { SCEWRAT } \\
\text { MCLRAT } \\
\text { HCDRAT }\end{array}$ & $\begin{array}{c}13 \cdot 5 \\
1 \cdot 35 \\
0 \cdot 250 \\
53 \cdot 3 \\
77 \cdot 9 \\
416 \cdot 4 \\
42 \cdot 1 \\
23 \cdot 1 \\
373 \cdot 6 \\
25 \cdot 2 \\
123 \cdot 8 \\
3966 \cdot 4 \\
1.84 \\
1.11 \\
1.78\end{array}$ & $\begin{array}{c}11 \cdot 0 \\
1 \cdot 51 \\
0 \cdot 355 \\
63 \cdot 0 \\
96 \cdot 2 \\
521 \cdot 0 \\
29 \cdot 9 \\
24 \cdot 8 \\
430 \cdot 9 \\
44 \cdot 1 \\
167 \cdot 1 \\
4440 \cdot 6 \\
1 \cdot 19 \\
1.23 \\
1.08\end{array}$ & $\begin{array}{c}11 \cdot 1 \\
1 \cdot 42 \\
0 \cdot 336 \\
59 \cdot 1 \\
94 \cdot 7 \\
559 \cdot 2 \\
28 \cdot 2 \\
25 \cdot 3 \\
461 \cdot 6 \\
35 \cdot 2 \\
179 \cdot 0 \\
5014 \cdot 2 \\
1 \cdot 12 \\
1.21 \\
1.04\end{array}$ & $\begin{array}{c}11.4 \\
1.62 \\
0.427 \\
65 \cdot 5 \\
91.9 \\
555.9 \\
24 \cdot 2 \\
24 \cdot 3 \\
423.5 \\
47 \cdot 5 \\
169.0 \\
4380.7 \\
0.97 \\
1.33 \\
0.91\end{array}$ & $\begin{array}{c}15 \cdot 3 \\
1 \cdot 43 \\
0 \cdot 246 \\
48 \cdot 1 \\
76 \cdot 4 \\
456 \cdot 8 \\
42 \cdot 6 \\
23 \cdot 3 \\
408 \cdot 1 \\
20 \cdot 3 \\
132 \cdot 3 \\
4387 \cdot 6 \\
1.94 \\
1 \cdot 13 \\
1.93\end{array}$ \\
\hline
\end{tabular}

Table 4 Mean cell density in horizontal bands

\begin{tabular}{|c|c|c|c|c|c|}
\hline Bands & Controls & $\begin{array}{l}\text { Ulcerative } \\
\text { colitis }\end{array}$ & $\begin{array}{l}\text { Chronic Crohn's } \\
\text { disease }\end{array}$ & Proctitis & $\begin{array}{l}\text { Small bowel } \\
\text { Crohn's disease }\end{array}$ \\
\hline $\begin{array}{l}\text { Superficial } \\
\text { Middle } \\
\text { Basal } \\
\text { HCDRAT }\end{array}$ & $\begin{array}{c}7834.5 \\
5616.5 \\
4480 \cdot 5 \\
1.78\end{array}$ & $\begin{array}{l}8633 \cdot 1 \\
8942 \cdot 4 \ddagger \\
8599 \cdot 2 \ddagger \\
1.08 \ddagger\end{array}$ & $\begin{array}{r}10146 \cdot 2^{*} \\
10620 \cdot 2 \ddagger \\
9981.8 \ddagger \\
1.04 \ddagger\end{array}$ & $\begin{array}{r}11644.8 \dagger \\
12708.9 \ddagger \\
12923.0 \ddagger \\
0.93 \ddagger\end{array}$ & $\begin{array}{r}8828 \cdot 1 \\
6730.6 \\
4970 \cdot 5 \\
1.93\end{array}$ \\
\hline
\end{tabular}

Significance of difference from the control group: ${ }^{*} p<0.01 ; \nmid p<0.005 ; \neq p<0.001$.

final outcome, six patients ultimately diagnosed with inflammatory bowel disease were classified as such by the analysis, and four were classified as normal. Of these four, two were in complete remission at the time of biopsy and one was a patient with Crohn's colitis but no evidence of rectal disease. Twenty of 24 patients who showed no evidence of inflammatory bowel disease, even after follow up, were classified as normal. Four patients in this group were classified as having inflammatory bowel disease. Overall, this indicates an accuracy in the diagnosis of normality or inflammatory bowel disease, which was active at the time and site of biopsy, of $85 \%$ in group F. None had been diagnosed by conventional examination.

\section{Discussion}

As far as we know, this study is the first to describe quantitatively both the inflammatory cell populations $\mathrm{N}$ and the dimensions and architecture of normal large bowel mucosa and that of chronic inflammatory bowel $N$ disease. As far as we know it is also the first to use a statistical approach to such combined data to define 0 properly the normal range, to investigate the contribution of cell counts (including plasma cells, and size, $\stackrel{\overparen{D}}{\mathbb{D}}$ shape and relation measurements) to the diagnosis of $\stackrel{\oplus}{?}$ inflammatory bowel disease, and to assess the value of 0 these measurements in the accurate diagnosis of borderline inflammatory changes in rectal biopsy specimens. 
Table 5 Stepwise discriminant analysis: controls compared with those with combined inflammatory bowel disease

\begin{tabular}{llll}
\hline & \multicolumn{3}{l}{ No of cases classified as: } \\
\cline { 2 - 4 } & Normal & $\begin{array}{l}\text { Inflammatory } \\
\text { bowel } \\
\text { disease }\end{array}$ & $\begin{array}{l}\text { \% } \\
\text { correct }\end{array}$ \\
\hline $\begin{array}{l}\text { Controls } \\
\text { Inflammatory }\end{array} \quad 19$ & 1 & $95 \cdot 0$ \\
$\begin{array}{l}\text { bowel disease } \\
\text { Small bowel }\end{array}$ & 3 & 49 & $94 \cdot 2$ \\
$\begin{array}{l}\text { Crohn's } \\
\text { Unknown }\end{array}$ & 8 & 0 & \\
Total & 24 & 10 & $94 \cdot 4$ \\
\hline
\end{tabular}

Variables entered: HCDRAT, SCEWRAT, MLS.

The application of stepwise discriminant analysis to the wide range of combinations of multiple counts and basic morphometric measurements in the different groups in this study consistently identified three major discriminants. These were measures of lamina propria cellularity and distribution of cells within the lamina propria, measurement of surface length to mucosal length ratio (MLS), and changes in surface epithelial cell and crypt epithelial cell height. Using a discriminant function based on the most sensitive indicator of change in cellularity of the lamina propria, together with MLS and the ratio of the surface cell height to crypt epithelial cell height (SCEWRAT), a high level of diagnostic accuracy was achieved from a few measurements with a $95 \%$ accurate separation of known cases and an $85 \%$ accurate separation of borderline cases which had presented previous difficulties to experienced diagnostic histopathologists.

A previously unsuspected finding was that in the normal large bowel mucosa, lamina propria cellularity is maximal in the zone immediately beneath the surface epithelium, and the increase in cellularity in chronic inflammatory bowel disease was detected most sensitively as an increase in cells in the basal third of the lamina propria. This was found to occur even in mild chronic inflammatory bowel disease, which might otherwise be regarded as of borderline or uncertain importance, and emerged as the most powerful single discriminant of chronic inflammatory bowel disease.

Crude measurement of total lamina propria cellularity (CELLEN) provided a good but only partial discrimination between normal and inflammatory bowel disease with, overall, $86 \%$ of known cases being correctly classified. Cellularity as judged by conventional histological examination has previously been shown to be of high predictive value for chronic inflammatory bowel disease. ${ }^{2}$ Counts of lamina propria cells in 10 consecutive areas and measurements of crypt diameter, interglandular distance, and numbers of glands in haematoxylin and eosin stained sections have been reported by Rubio et $a l$ in normal rectal mucosa and chronic colitis, and shown to be of diagnostic value. ${ }^{89}$ Although we previously reported a pronounced difference in plasma cell counts between normal and chronically inflamed rectal mucosa and a positive correlation between plasma cell counts and overall severity of inflammation, ${ }^{6}$ in this investigation we did not find that specific isotype plasma cell counts offered any additional discriminant value in the diagnosis of chronic colitis over other measurements. There is a wide normal range in plasma cell counts that overlaps with the lower range of counts in inflammatory bowel disease. In spite of some claims for the diagnostic value of plasma cell counts in detecting acute bacterial colotis $^{10}$ and the differential diagnosis of chronic colitis ${ }^{11}$ this overlap limits their use as sole discriminant in practical diagnosis. Our findings did not support previous suggestions of subtle quantitative abnormalities at remote mucosal sites in patients with Crohn's disease elsewhere. ${ }^{12}$

All the cellularity measurements showed appreciable covariation, and to study their true relative value in separating cases it was necessary to work interactively and run repeat stepwise discriminant programs suppressing dominant covariables. Taking account of this, the most remarkable finding was the very high level of discrimination between normal and known chronic inflammatory bowel disease, and even within the borderline group when refinements of cellularity measurements were made to take account of the change in cell distribution within the lamina propria between normal and chronically inflamed mucosa. The ratio of counts in the superficial and basal thirds (HCDRAT) emerged as the strongest discriminant.

Mucosal morphometry by automatic image analysis has previously shown some discriminant factors for types of inflammatory bowel disease including the surface to crypt cell height ratio (SCEWRAT), ${ }^{13}$ but the measurements of mucosal size and architecture that emerged in this study were of less discriminant value than the cellularity. The surface:length ratio (MLS) which also emerged is a measure of surface contour. An increase in this value in chronic inflammatory bowel disease is a reflection of a villous surface resulting from separation of crypts yielding a contoured surface with broad villous-like projections. This has already been identified subjectively as highly discriminant. ${ }^{2}$

This study shows that mathematical analysis of quantitative histological data can be used to investigate the classification of inflammatory changes in biopsy specimens and to define more precisely the limits of normality and abnormality. It also shows that this approach can identify ways in which a group of borderline cases, previously undiagnosable by conventional histological examination, can be accurately 


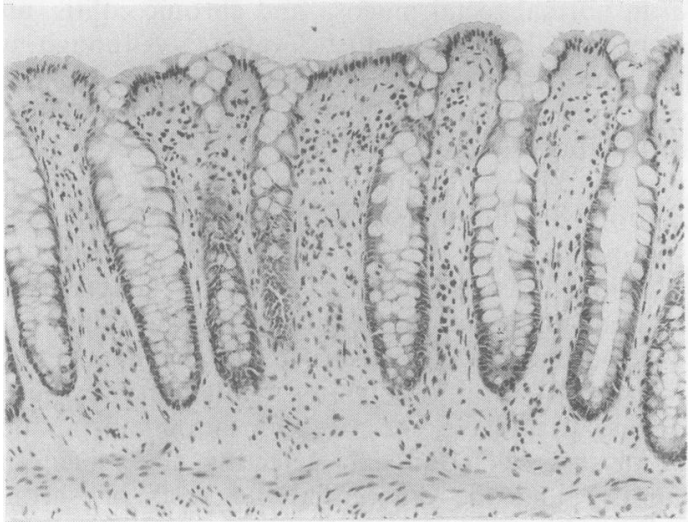

Fig 2 Normal rectal mucosa showing concentration of cells in lamina propria close to surface.

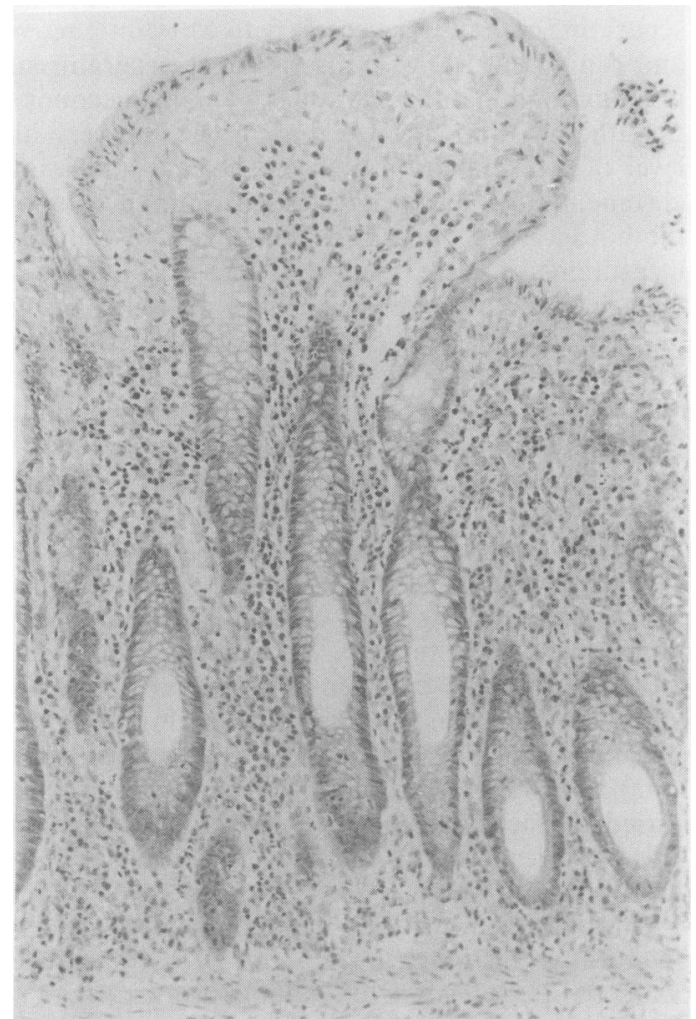

Fig 3 Rectal mucosa in florid chronic inflammatory bowel disease. Section shows pronounced irregularity of surface, sharp increase in cellularity throughout lamina propria with similar density in superficial and basal zones, and a reduction in ratio of surface epithelial height:crypt epithelial height. classified. Discriminant analysis was selected as being particularly useful when the endpoint was not precisely defined, as in the case of the borderline group, $\overline{0}$ because it uses a mathematical model of probability density. Used interactively it can assist in the selection of measurements for a diagnostic system. The selec- $\overline{0}$ tion of appropriate measurements is an important part 음 of designing this type of study, and interaction with the $\frac{\bar{\omega}}{\bar{D}}$ initial results of the analysis is essential to develop the $\stackrel{\oplus}{\circ}$ best discriminant system. This becomes especially important when trying to relate measured changes to conventional subjective interpretation, as the eye is . very poor at judging absolute counts and

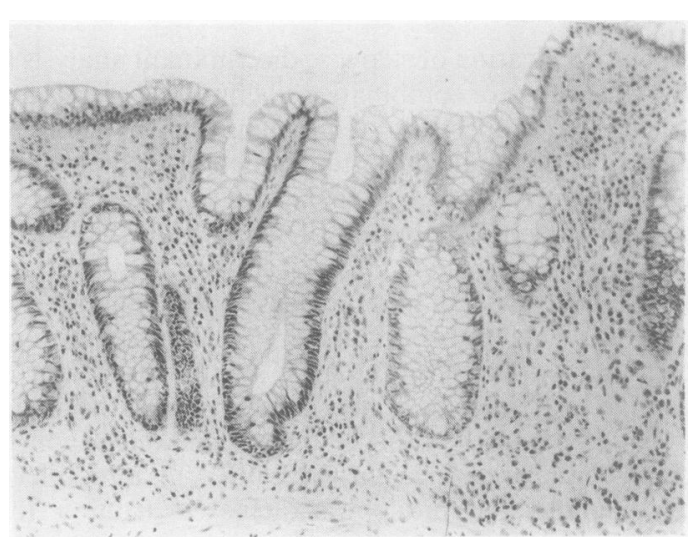

Fig 4 Rectal mucosa in mild chronic inflammatory bowel disease. There is some surface irregularity and even increase in chronic inflammatory cells throughout lamina propria. Changes in surface epithelium are slight.

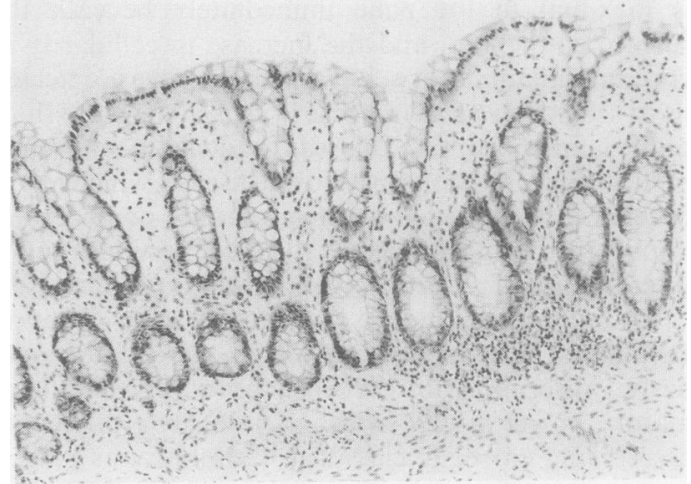

Fig 5 Rectal biopsy specimens initially classified as "borderline", but subsequently shown to be chronic inflammatory bowel disease. There is some surface irregularity, an increase of cellularity which is best distinguished from the normal range in the basal third of the lamina propria and by comparison of this with the superficial third. 
measurements but can give very sensitive discrimination of relative size.

Such an exercise as this is an essential preliminary to developing a framework for more reproducible diagnostic histopathology, whether the aim is to produce a computerised "expert system" for automated diagnosis using image analysis or to improve the accuracy and reproducibility of conventional diagnostic approaches. This study has implications for both. All three features identified by discriminant analysis can be appreciated in conventional subjective histological examination. In the normal rectal mucosa most chronic inflammatory infiltration is seen immediately beneath the surface epithelium, with relatively sparse cellular infiltration around the bases of crypts and adjacent to the muscularis mucosae (fig 2 ). A redistribution of the infiltrating cells such that there is a similar density in the basal third to that of the superficial third is seen in frank chronic colitis (fig 3), but also in mild chronic colitis (fig 4), and the importance of recognising an increase in cellularity in the basal third can also be seen in biopsy specimens from patients who have subsequently confirmed chronic inflammatory bowel disease, in which the total increase in cellularity is minimal (fig 5). In the normal large bowel mucosa the surface epithelial cell height exceeds the height of the crypt epithelium (fig 2), but this is lost or reversed in chronic inflammatory bowel disease to a variable degree. It is easily appreciated subjectively in florid colitis (fig 3 ), but is less easy to judge unaided when of minor degree (figs 4 and 5). The application of the criteria identified by the study to routine examination of rectal biopsy specimens can offer improvements in the precision of subjective diagnosis.

This study has shown that it is possible to achieve very high levels of diagnostic accuracy in the detection of chronic inflammatory bowel disease in rectal biopsy specimens using quantitative and statistical methods, even when it had not been possible to make a definitive, subjective diagnosis previously. This offers great potential for the use of image analysis and mathematical methods to improve considerably the accuracy and reproducibility of histopathological diagnosis. At present, however, the application of automated diagnosis is limited by the poor performance of current automatic image analysis in counting small particles in histological sections. Without the ability to measure the density and distribution of an inflammatory infiltrate, image analysis is limited in what it can offer in the study of inflammatory bowel disease. The use of a semiautomatic digitising system probably requires too much expertise for satisfactory use in routine diagnosis and can be time consuming. The possibility of developing a simplified scoring system and its comparison with the subjective applica- tion of the criteria remains to be evaluated. Three easily recognisable histological features have been statistically validated as offering the potential to detect accurately inflammatory bowel disease and distinguish even very mild disease from normal. The change in distribution of inflammatory cells with a pronounced increase in the deep level of the lamina propria and a change in the ratio of cell density between superficial and deep levels from around 1.8:1 to 1:1 are new findings with the potential for detecting minor, but clinically important, degrees of inflammation.

We are very grateful to the National Association for Colitis and Crohn's Disease for generous financial support. We also thank Mr B Thornton and Mr PM Stephenson for technical help, Mr R Yabsley for photography, and Dr Brian Newman, department of statistics, University College, London, for statistical advice.

\section{References}

1 Jones JH, Lennard-Jones JE, Morson BC, et al. Numerical taxonomy and discriminant analysis applied to non-specific colitis. $Q J$ Med 1973;42:715-32.

2 Surawicz CM, Belic L. Rectal biopsy helps to distinguish acute self-limited colitis from idiopathic inflammatory bowel disease. Gastroenterology 1984;86:104-13.

3 Elliott PR, Lennard-Jones JE, Bartram CI, et al. Colonoscopic diagnosis of minimal change colitis in patients with a normal sigmoidoscopy and normal air-contrast barium enema. Lancet 1982;ii:650

4 Kingham JGC, Levison DA, Ball JA, Dawson AM. Microscopic colitis-a cause of chronic watery diarrhoea. $\mathrm{Br} M e d \mathrm{~J}$ 1985;285:1601-4.

5 Surawicz CM, Goodell SE, Quinn TC, et al. Spectrum of rectal biopsy abnormalities in homosexual men with intestinal symptoms. Gastroenterology 1986;91:651-9.

6 Scott BB, Goodall A, Stephenson PM, Jenkins D. Rectal mucosal plasma cells in inflammatory bowel disease. Gut 1983;24: 519-24.

7 Jenkins D, Goodall A, Gillet FR, Scott BB. Defining duodenitisa quantitative histological study of the mucosal responses and their correlations. J Clin Pathol 1985;38:1119-26.

8 Rubio CA, Kock Y. A digital quantitative method of estimating inflammation in the rectal mucosa. 1. The normal limits in asymptomatic patients. Scand J Gastroenterol 1981;16:731-5.

9 Rubio CA, Johansson C, Uribe A, Kock Y. A quantitative method of estimating inflammation in the rectal mucosa. IV. Ulcerative colitis in remission. Scand J Gastroenterol 1984;19:525-30.

10 Van Spreeuwel JP, Lindeman J, Meijer CJLM. A quantitative study of immunoglobulin containing cells in the differential diagnosis of acute colitis. J Clin Pathol 1985;38:774-7.

11 Rosekrans PCM, Meijer CJLM, Van der Wal AM, Cornelisse CJ, Lindeman J. Immunoglobulin containing cells in inflammatory bowel disease of the colon: a morphometric and immunohistochemical study. Gut 1980;21:941-7.

12 Ferguson R, Allan RN, Cooke WT. A study of the cellular infiltrate of the proximal jejunal mucosa in ulcerative colitis and Crohn's disease. Gut 1975;16:205-8.

13 Thompson EM, Price AB, Altman DG, et al. Quantitation of inflammatory bowel disease using computerised interactive image analysis. J Clin Pathol 1985;38:631-8.

Requests for reprints to: Dr D Jenkins, Department of Histopathology, Whittington Hospital, Highgate Hill, London N19 5NF, England. 